\title{
Fluorine abundances and the puzzle of globular cluster chemical history ${ }^{\star}$
}

\author{
P. de Laverny and A. Recio-Blanco
}

\author{
Laboratoire Lagrange (UMR 7293), Université de Nice Sophia Antipolis, CNRS, Observatoire de la Côte d'Azur, BP 4229, \\ 06304 Nice Cedex 4, France \\ e-mail: laverny@oca.eu
}

Received 18 March 2013 / Accepted 29 May 2013

\section{ABSTRACT}

\begin{abstract}
Aims. A few studies have already shown that the abundance of fluorine in a few galactic globular clusters is strongly varying from star-to-star. These unexpected chemical properties are an additional confirmation of the chemical inhomogeneities already found in several globular clusters, and probably caused by the first generations of stars formed in these systems. The aim of this article is to complement our understanding of the fluorine behaviour in globular cluster stars and to look for new constraints on the formation histories of their multiple stellar populations.

Methods. We have collected near-IR spectra with VLT/CRIRES of 15 red giant branch stars belonging to four different globular clusters spanning a wide range of metallicity: 47 Tuc, M 4, NGC 6397 and M 30. We have estimated the fluorine abundance of these targets from the spectral synthesis of one HF line at $2.336 \mu \mathrm{m}$. Sodium and iron abundances have also been determined from two individual lines of FeI and NaI found in the same spectra.

Results. No anticorrelation between fluorine and sodium abundances are found for the most metal-rich cluster of the sample (47 Tuc). In this cluster, RGB stars indeed exhibit rather small differences in $[\mathrm{F} / \mathrm{Fe}]$ unlike the larger ones found for the $[\mathrm{Na} / \mathrm{Fe}] \mathrm{ratios}$. This reveals a rather inhomogeneous stellar system and a complex chemical evolution history for 47 Tuc. In M 4, one star of our study confirms the previous Na-F distribution reported by another group in 2005. For the two very metal-poor globular clusters (NGC 6397 and M 30), only upper limits of fluorine abundances have been derived. We show that F-abundances could be estimated (but with high uncertainty) in such metal-poor globular clusters with current telescopes and spectrographs only if unexpected F-rich giants are found and/or exceptional observational conditions are met.

Conclusions. The distribution of the fluorine and sodium abundances in globular clusters reveal that their RGB members seem to belong to two well-separated regions. All the RGB stars analysed so far in the different globular clusters are indeed found to be either F-rich Na-poor or F-poor Na-rich. Such well-separated bimodal regimes are consistent with the separate formation episodes suspected in most galactic globular clusters.
\end{abstract}

Key words. globular clusters: general - stars: abundances - globular clusters: individual: 47 Tuc - globular clusters: individual: M 4 globular clusters: individual: NGC 6397 - globular clusters: individual: M 30

\section{Introduction}

It is now well admitted that galactic globular clusters (GC) have a formation history that is much more complex than previouly thought. Several signatures of successive stellar populations have indeed been reported in most GCs (see Gratton et al. 2012, for an extensive review). However, the different episodes of stellar formation in these clusters are still not well understood. One possible way to disentangle this problem is to get information on the chemical evolution history of each GC. The chemical signatures of the previous populations can indeed be searched at the surface of the present stars in these clusters. Up to now, two main ranges of mass for the first-generation polluting stars in GCs have been favoured: intermediate-mass on the asymptotic giant branch (AGB) or fast-rotating massive stars on the main sequence (see for instance, Charbonnel et al. 2013; Renzini 2013).

A very interesting chemical element for getting a better view of GC multiple stellar populations is fluorine. Indeed, although the exact origin of this chemical specy in the Universe is still an open question, there are only two main ranges of stellar masses

* Based on observations collected with the CRIRES spectrograph at the VLT/UT1 Antu telescope (Paranal Observatory, ESO, Chile), Programs 081.D-0276 \& 083.D-0416. in which it is suspected to be produced: massive stars (type II supernovae and/or Wolf-Rayet stars) or low-mass stars on the AGB (see Recio-Blanco et al. 2012, for a more detailed description of these production mechanisms). However, from an observational point of view, there is direct evidence of fluorine production only in low-mass AGB stars (Jorissen et al. 1992; Abia et al. 2009, 2010; Recio-Blanco et al. 2012). In contrast, two main types of stars are known to destroy fluorine: fast-rotating massive stars (Prantzos et al. 2007) and intermediate-mass AGBs that experience hot-bottom burning process, which is also a phase of sodium production. Therefore, exploring the $\mathrm{F}$ and $\mathrm{Na}$ content in GC may help disentangle the formation history of these clusters.

Unfortunately, the abundance of fluorine in globular cluster stars has still not been explored or understood very well. Up to now, F-abundances have been reported for very few stars found in only four different GCs. The first fluorine abundances in GC stars were determined by Cunha et al. (2003) for two red giant stars (RGB) of $\omega$ Cen. These stars belong to the metal-rich component of this GC (their metallicity is $[\mathrm{Fe} / \mathrm{H}]=$ -0.9 and $-1.2 \mathrm{dex}$ ), and actually, only an upper limit of the F-abundance has been derived for one of them. The other RGB target in $\omega$ Cen exhibits a moderate fluorine content $([\mathrm{F} / \mathrm{Fe}]=$ -0.2 dex). Then, Smith et al. (2005) published the F-content 
Table 1. Atmospheric parameters and derived chemical abundances for stars in our sample.

\begin{tabular}{|c|c|c|c|c|c|c|c|c|c|c|c|}
\hline & {$[\mathrm{Fe} / \mathrm{H}]^{a}$} & {$[\alpha / \mathrm{Fe}]^{a}$} & Star & $K$ (mag) & $T_{\text {eff }}(\mathrm{K})$ & $\log (g)\left(\mathrm{cm} \mathrm{s}^{-2}\right)$ & $V_{\text {micro }}\left(\mathrm{km} \mathrm{s}^{-1}\right)$ & {$[\mathrm{Fe} / \mathrm{H}]^{c}$} & {$[\mathrm{Na} / \mathrm{Fe}]$} & $A(\mathrm{~F})^{b}$ & {$[\mathrm{~F} / \mathrm{Fe}]$} \\
\hline Arcturus & -0.52 & +0.31 & & & 4286 & 1.66 & 1.74 & -0.50 & $+0.23^{c}$ & 4.16 & $+0.10^{c}$ \\
\hline \multirow[t]{6}{*}{47 Tuc } & -0.76 & +0.3 & \#41806 & 7.36 & 3500 & 0.1 & 2.6 & -0.98 & $+0.01^{c}$ & 3.15 & $-0.43^{c}$ \\
\hline & & & \#68261 & 7.93 & 3800 & 0.6 & 2.3 & -0.90 & $+0.23^{c}$ & 3.4 & $-0.26^{c}$ \\
\hline & & & $\# 56265$ & 8.49 & 3880 & 0.8 & 2.3 & -0.80 & $+0.13^{c}$ & 3.5 & $-0.26^{c}$ \\
\hline & & & \#68039 & 8.78 & 4015 & 1.0 & 2.1 & -0.90 & $+0.53^{c}$ & $<3.5$ & $<-0.16^{c}$ \\
\hline & & & \#38841 & 8.93 & 4015 & 1.0 & 2.1 & -0.90 & $+0.23^{c}$ & 3.5 & $-0.16^{c}$ \\
\hline & & & \#86622 & 8.99 & 4020 & 1.0 & 2.1 & -0.95 & $+0.43^{c}$ & 3.6 & $-0.11^{c}$ \\
\hline \multirow[t]{3}{*}{ M 4} & -1.18 & +0.4 & \#45330 & 8.16 & 4145 & 1.3 & 2.0 & -1.10 & $+0.03^{c}$ & 3.4 & $-0.06^{c}$ \\
\hline & & & \#38229 & 9.28 & 4360 & 1.8 & 1.9 & & $+0.31^{d}$ & $<4.0$ & $<+0.62^{d}$ \\
\hline & & & \#39797 & 10.07 & 4375 & 2.1 & 1.8 & & $+0.11^{d}$ & $<4.0$ & $<+0.62^{d}$ \\
\hline \multirow[t]{4}{*}{ NGC 6397} & -1.99 & +0.4 & $\# 73589$ & 7.89 & 4490 & 1.2 & 1.7 & $<-1.5$ & $+0.12^{d}$ & $<3.0$ & $<+0.43^{d}$ \\
\hline & & & $\# 73212$ & 8.43 & 4565 & 1.4 & 1.7 & $<-1.5$ & $<-0.08^{d}$ & $<3.3$ & $<+0.73^{d}$ \\
\hline & & & $\# 51362$ & 8.52 & 4640 & 1.5 & 1.6 & $<-1.5$ & $+0.02^{d}$ & $<3.5$ & $<+0.93^{d}$ \\
\hline & & & \#52830 & 8.54 & 4630 & 1.5 & 1.6 & & $+0.12^{d}$ & $<3.7$ & $<+1.13^{d}$ \\
\hline \multirow[t]{2}{*}{ M 30} & -2.33 & +0.4 & \#3998 & 8.86 & 4150 & 0.5 & 2.0 & & $+0.16^{d}$ & $<2.6$ & $<+0.37^{d}$ \\
\hline & & & \#7640 & 9.77 & 4445 & 0.9 & 1.8 & & $+0.46^{d}$ & $<3.2$ & $<+0.97^{d}$ \\
\hline
\end{tabular}

Notes. ${ }^{(a)}$ These columns refer to the mean $[\mathrm{Fe} / \mathrm{H}]$ ratios of each cluster (Carretta et al. 2009) and the corresponding $[\alpha / \mathrm{Fe}]$ ratios as seen in most galactic stars for a given $[\mathrm{Fe} / \mathrm{H}]$. For Arcturus, the stellar parameters and $[\alpha / \mathrm{Fe}]$ are from Ramírez \& Meléndez $(2005){ }^{(b)}$ For the chemical abundances, we use the classical relation $A(\mathrm{~F})=\log [n(\mathrm{~F}) / n(\mathrm{H})]+12$. The adopted $\mathrm{F}$ solar abundance is 4.56 . $^{(c)}$ These chemical ratios are computed with the iron abundance measured with the FeI line at $2.3308 \mu \mathrm{m}$. The adopted Na and Fe solar abundances are 6.17 and 7.45 , respectively. ${ }^{(d)}$ These chemical ratios are computed with the mean iron abundance of each cluster given in Col. 2.

of seven giant stars of the GC M 4, which mean metallicity is $[\mathrm{Fe} / \mathrm{H}]=-1.18$ dex (Carretta et al. 2009). This fundamental work has shown that the fluorine abundances strongly vary from star-to-star ([F/Fe] is varying from $\sim-0.75$ to $\sim-0.1$ dex) and that they are anticorrelated with sodium abundances. This leads Smith et al. (2005) to suggest that a link may exist between the F-abundances and the chemical pollutions caused by previous stellar generations in M 4 in which intermediate-mass AGB stars might have played a major role. At similar metallicity, Yong et al. (2008) have studied the fluorine content in five RGBs of the GC NGC $6712([\mathrm{Fe} / \mathrm{H}]=-1.0 \mathrm{dex})$. They again found a large scatter in the F-abundances: $[\mathrm{F} / \mathrm{Fe}]$ is varying from -1.0 to $-0.17 \mathrm{dex}$. The analysed stars can be grouped into two different regimes: two stars are found to be F-poor and three are F-rich. Such a picture could be interpreted, as in M 4, with the yields of intermediate-mass AGB and/or those of massive first-generation stars. Finally, fluorine abundances have also been studied in the more metal-poor massive GC M 22 with a mean metallicity $[\mathrm{Fe} / \mathrm{H}] \sim-1.7$ dex and a dispersion around 0.2 dex. Alves-Brito et al. (2012) derived F-abundances in five RGB stars and upper limits in two other stars of this cluster. No correlation between $\mathrm{F}$ and $\mathrm{O}$ or $\mathrm{Na}$ abundances is seen by these authors. This conclusion has been ruled out by D'Orazi et al. (2013), who tried to derive fluorine abundances in six RGB of M 22 (four of them in common with Alves-Brito et al. 2012). It is shown that the telluric substraction inherent to any $K$-band spectroscopy may partly explain the largest differences in the F-abundances derived by both groups. However, these rederived F-abundances in M 22 can still be questioned (see our discussion on F-abundance determination in metal-poor GC stars in Sect. 4.2), and a deeper analysis of these spectra reveal that no definitive conclusions on the $\mathrm{F}$ and $\mathrm{Na}$ distributions in M 22 stars can be drawn (see de Laverny et al., in prep.).

To provide new constraints about the nature of the firstgeneration stars that played a major role in the chemical evolution of globular clusters, we present in this work the fluorine abundances (or upper limits) of 15 RGB stars belonging to four GC spanning a wide range of metallicity (from $[\mathrm{Fe} / \mathrm{H}]=-0.76$ to $-2.33 \mathrm{dex}$ ). This paper is organised as follows: Sect. 2 is devoted to the description of the observations, the selection of the targets, the determination of their stellar parameters, and chemical analysis. We then present our abundance determinations in the most metal-rich clusters studied in Sect. 3. Our results are discussed in Sect. 4 with a particular emphasis on the limitations of the F-abundance determinations in metal-poor GCs. Finally, Sect. 5 summarizes our conclusions.

\section{Observations and chemical analysis}

\subsection{CRIRES observations and targets selection}

The spectra were collected with the VLT/CRIRES spectrograph during two nights in July 2008 (Program 081.D-0276) and in service mode one year later (Program 083.D-0416). We adopted the CRIRES standard setup WLEN.ID $=24 /-1 / i$ that allows the observation of the HF(1-0) R9 line at $\sim 2.336 \mu$ m on which our abundance analysis is based.

The selected targets are giant stars located close to the tip (or slightly below) of the RGB of four globular clusters. Bright RGB stars have been favoured since the HF line appears stronger in cooler stars. We also observed slightly less bright stars in order to explore the possibility of detecting the F line in hotter but possibly more F-enriched stars and to avoid the possible confusion between RGB and AGB stars where fluorine could be synthetized. To explore the variation in fluorine between different chemical environments, we also selected GC spanning a wide range of metallicities: metal-rich (47 Tuc), intermediate metallicity (M 4), and very metal-poor GC (NGC 6397 and M 30). The RGB stars of these clusters have been selected from the colour-magnitude diagrams of Rosenberg et al. (2000) for M 4 and NGC 6397, Momany et al. (2004) for M 30 and Momany (priv. comm.) for 47 Tuc. These works provide the BVI photometry of the selected targets, and we also collected their 2MASS $J H K$ magnitudes. The observed stars are listed in Table 1 with the naming convention of their visible photometry together with their $K$-band magnitude. The GC membership of the selected 
targets was confirmed with the radial velocity derived from the CRIRES spectra. Together with these RGB stars and in order to remove the lines of the Earth's atmosphere superimposed on the stellar spectra, several telluric standards were observed at the same airmass as the science targets, just before and after them. The selected standards were featureless hot OB stars. The spectra were reduced with the ESO/CRIRES pipeline, and standard IRAF procedures were used to remove the telluric contributions.

\subsection{Stellar atmospheric parameters}

We first assigned to every star of each cluster the mean cluster metallicty estimated by Carretta et al. (2009), together with an $\alpha$-element enhancement with respect to iron $([\alpha / \mathrm{Fe}])$ as found in most galactic GC stars for a given $[\mathrm{Fe} / \mathrm{H}]$. Then, the effective temperature of each star has been computed as the mean of the temperatures estimated from the $(V-J)$ and $(V-K)$ colours following the calibration for giant stars of Ramírez \& Meléndez (2005, their Eq. (2)). The differences between these two temperature estimates are always less than $\sim 60 \mathrm{~K}$ and less than $\sim 15 \mathrm{~K}$ for $75 \%$ of the sample. When computing these $T_{\text {eff }}$ values, we adopted the extinction from the GC foreground reddening reported by Harris (1996, revised in 2010) and from the relations given in Cardelli et al. (1989). We point out that the differential reddening present in the direction of the GC M 4 does not strongly affect our $T_{\text {eff }}$ estimates since we mostly rely on the IR photometry of the targets. The two estimates of $T_{\text {eff }}$ for the three targets of M 4 indeed vary by less than $\pm 40 \mathrm{~K}$. The surface gravities were calculated from the derived effective temperatures, the dereddened magnitudes, the bolometric correction estimated from the relation provided by Alonso et al. (1999), and the distance (Harris catalogue), and we adopted a typical mass of $0.8 M_{\odot}$ for the RGB stars in our sample. A slightly heavier mass of $0.9 M_{\odot}$ would increase $\log (g)$ by only $0.05 \mathrm{dex}$, and would have a negligible effect on the derived abundances (see below the discussion on the error analysis). The error on the other parameters used for the surface gravity estimates can also be neglected since their impact on the derived $\log (g)$ is very weak. Finally, the microturbulent velocity was estimated from the empirical relation for metal-poor giant stars derived by Pilachowski et al. (1996).

We have searched the literature for checking our atmospheric parameter estimation. One of our targets (\#51362 in NGC 6397) has already been studied in detail by Lind et al. (2011). The differences between their adopted atmospheric parameters and ours are only $+87 \mathrm{~K}, 0.02 \mathrm{dex}$, and $0.01 \mathrm{~km} \mathrm{~s}^{-1}$ for $T_{\text {eff }}, \log (g)$, and $V_{\text {micro }}$, repectively. Moreover, our brightest target in the GC M 4 (\#45330) can be compared to the faintest one (\#L3413) of Smith et al. (2005), which both have very close $T_{\text {eff }}$ (within $30 \mathrm{~K}$ ). Although the stellar parameters are derived from different methods (spectroscopic versus photometric), they do agree: within 0.1 dex for the surface gravity and $0.35 \mathrm{~km} \mathrm{~s}^{-1}$ for $V_{\text {micro }}$. These different checks confirm the validity of our procedure in estimating the stellar atmospheric parameters adopted for the chemical analysis. All these parameters are reported in Table 1.

\subsection{Chemical analysis}

The fluorine abundances were derived from the unblended $\mathrm{HF}(10) \mathrm{R} 9$ line at $2.3358 \mu \mathrm{m}$. We have shown in Abia et al. (2009) that this line is the best F indicator in this spectral range. This domain also contains several $\mathrm{CO}$ lines, together with a nonblended NaI line at $2.3379 \mu \mathrm{m}$ and a FeI line at $2.3308 \mu \mathrm{m}$

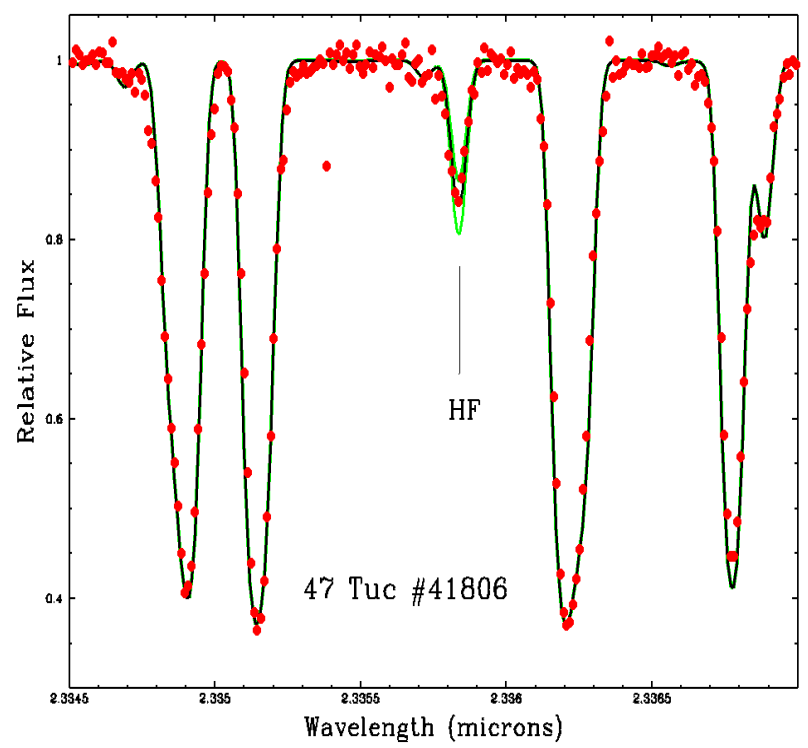

Fig. 1. Observed (filled red circles) and synthetic spectra (continuous lines) around the HF(1-0) R9 line for the star 47 Tuc \#41806. The best fit (black line) corresponds to the fluorine abundance reported in Table 1 and synthetic spectra computed with \pm 0.1 dex changes in the F-abundance are superimposed (green lines).

that have been used for this chemical analysis. We adopt the same linelist as in our previous works (see Abia et al. 2009; Recio-Blanco et al. 2012). The theoretical spectra were computed with the TurboSpectrum code (Alvarez \& Plez 1998, and further improvements by Plez). Spherical MARCS model atmospheres (Gustafsson et al. 2008) with standard chemical composition (i.e. with $\alpha$ enhancements typical of the low-metallic clusters under study), a microturbulent parameter of $2 \mathrm{~km} \mathrm{~s}^{-1}$ and a mass of $1 M_{\odot}$ were adopted. For the different stars under study (see Table 1), interpolations at their estimated stellar atmospheric parameters were performed. Solar abundances are those adopted by Gustafsson et al. (2008). We also assumed a carbon isotopic ratio ${ }^{12} \mathrm{C} /{ }^{13} \mathrm{C}=5$ for all our targets, and we checked that this assumption does not affect our derived abundances. Finally, the synthetic spectra were broadened by convolution with a Gaussian profile of $F W H M=6 \mathrm{~km} \mathrm{~s}^{-1}$ to match the observed line widths. The derived F, Na, and $\mathrm{Fe}$ abundances (or the estimated upper limits) are reported in Table 1, and we show in Fig. 1 an example of an observed and synthetic spectrum for the brightest target of 47 Tuc.

This procedure for the chemical analysis was firstly checked by fitting the high-resolution IR spectrum of Arcturus (Hinkle et al. 1995) and adopting the stellar parameters and abundances of Ramírez \& Meléndez (2005). Our derived abundances are reported in Table 1 . These IR NaI and FeI abundances differ by +0.07 dex and -0.02 dex, respectively, with respect to those of Ramírez \& Meléndez (2005) that were derived from the optical domain (and considering that they adopted a solar iron abundance of 7.50, whereas we adopt 7.45 in the present work). We also found an abundance of fluorine in Arcturus of 4.16 (i.e. $[\mathrm{F} / \mathrm{Fe}]=+0.10$ ), in total agreement with our previous determination (Abia et al. 2009), although the adopted atmospheric parameters slightly differ since in the present work we favoured the Arcturus stellar parameters of Ramírez \& Meléndez (2005). Moreover, we also checked our abundance determinations with the Na-rich second generation star \#51362 in NGC 6397 studied by Lind et al. (2011). They report a sodium 
abundance $A(\mathrm{Na})=4.23$ that is very close to the value we measured $A(\mathrm{Na})=4.2$. Furthermore, their metallicity $[\mathrm{Fe} / \mathrm{H}]$ lies between -2.06 and -2.10 , and it is consistent with the upper limit we derived $(<-1.5)$.

On the other hand, we point out that the estimated chemical abundances reported in Table 1 reveal that the derived metallicity for each star of 47 Tuc is slightly more metal-poor than expected from the mean metallicity of this cluster (adopted from Carretta et al. 2009). Our mean $[\mathrm{Fe} / \mathrm{H}]$ is indeed about 0.14 dex lower than the Carretta et al. (2009) one, i.e. a difference larger than our estimated uncertainty that cannot be explained by the different adopted solar reference values. This could be explained by the fact that our $[\mathrm{Fe} / \mathrm{H}]$ derivations rely on only one FeI line and could be less robust because of telluric line contaminations that could alter the line profile and the continuous position. However, even if some iron abundance scatter is found from star-to-star within the present sample, this scatter is smaller than the estimated error and can thus be attributed entirely to the measurement uncertainties. The analysed iron line is therefore a rather good indicator of the stellar metallicity. This is also confirmed by the correct metallicity derived for Arcturus and by the rather good agreement found between the iron abundance derived by us in one star of M 4 and the Carretta et al. (2009) mean value. Therefore, the $[\mathrm{Fe} / \mathrm{H}]$ values derived from this line have been adopted in the present study when available.

Finally, we estimated that the typical errors on the derived abundances induced by uncertainties on the stellar atmospheric parameters are around $\pm 0.2 \mathrm{dex}, \pm 0.1 \mathrm{dex}$, and $\pm 0.1 \mathrm{dex}$ for fluorine, sodium, and iron, respectively. These estimates were computed as follows: for the target 47 Tuc \#68261 of Table 1 (which is typical of our sample), we re-estimated its abundances by varying its stellar parameters by $\Delta T_{\text {eff }}$ of $\pm 100 \mathrm{~K}, \Delta \log (g)$ of $\pm 0.2 \mathrm{dex}, \Delta[\mathrm{Fe} / \mathrm{H}]$ of $\pm 0.1 \mathrm{dex}$, and $\Delta V_{\text {micro }}$ of $\pm 0.5 \mathrm{~km} \mathrm{~s}^{-1}$. These different contributions were then summed in quadrature, together with the error in the fit (around $\pm 0.05 \mathrm{dex}$ ) dominated by the continuum placement and the telluric residuals. We point out that other possible systematic sources of uncertainties (as log gf uncertainties, for instance) are not taken into account in this error budget.

\section{Fluorine abundances in 47 Tuc and M 4}

We present in this section the estimated fluorine abundances in 47 Tuc and M 4 stars (i.e. the most metal-rich clusters of the present study). We refer to the next section for the discussion on the F-abundance upper limits derived for the two other studied GCs that are much more metal-poor (NGC 6397 and M 30).

\subsection{Tuc}

For the fluorine content of RGB stars in 47 Tuc, we illustrate in Fig. 2 the derived F-abundances versus the sodium ones. Although the error bars of each determination are large, we detect, as in all the other clusters studied so far, a large scatter in the $\mathrm{F}$ and $\mathrm{Na}$ abundances. The amplitude of these abundance variations exceeds the measurement uncertainties. The most fluorinerich star is found to be enriched by 0.45 dex with respect to the most F-poor one and their Na-content also differs by almost the same amount (but their iron content are quasi-identical). On the other hand, the range of the sodium abundances for these six stars of 47 Tuc is even broader and spans about 0.6 dex. 47 Tuc is thus a rather inhomogeneous cluster when one examines its $\mathrm{F}$ and $\mathrm{Na}$ chemical properties.

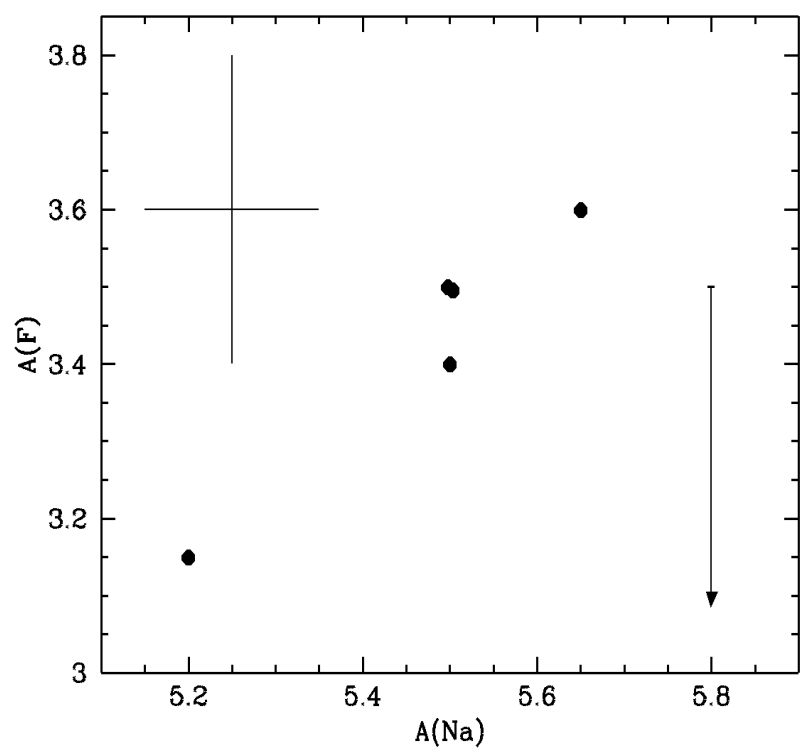

Fig. 2. Fluorine abundances with respect to sodium abundances for the RGB stars of 47 Tuc. For one star (\#68039), only an upper limit for fluorine has been derived. It is indicated with an arrow oriented towards low fluorine abundances. Within the abundance uncertainties, no (anti)correlation are detected between fluorine and sodium.

However, from this Fig. 2, no clear (anti-)correlation between the fluorine and sodium chemical abundances is seen with the present sample, unlike what has been reported in the other galactic globular cluster M 4 (see Fig. 3 and next subsection). Such an anticorrelation in 47 Tuc is mostly ruled out by two stars of the sample (\#41806 and \#86622). First, the RGB star \#41806 is indeed found to be very poor in $\mathrm{F}$ and $\mathrm{Na}$ (see its spectrum in Fig. 1). We have carefully checked this spectrum, the telluric contaminations, and the abundances derived for this star, and we are confident of them. Furthermore, although one possible source of uncertainty could be the estimation of the effective temperature, we have checked that adopting a different $T_{\text {eff }}$ would not reveal any Na-F anticorrelation in 47 Tuc. For instance, a hotter $T_{\text {eff }}$ would lead to a more enriched star in both fluorine and sodium, thus ruling out anyway a possible anticorrelation. Secondly, the other star that could be incompatible with a Na-F anticorrelation is the target \#86622. This RGB is indeed not only Na-rich but also very enriched in fluorine. It is actually the most F-rich star we have observed in 47 Tuc.

With the present sample, we are therefore confident that no anticorrelation between $\mathrm{F}$ and $\mathrm{Na}$ is detected in 47 Tuc. Increasing the size of the sample would perhaps help to confirm or not this conclusion. However, if an anticorrelation were found in the future, two stars in our study (representing one third of our total sample) would be rather exotic, and their specific $\mathrm{Na}$ and $\mathrm{F}$ properties should be understood.

\section{2. $M 4$}

For this globular cluster, our study adds a new point in the F-Na relation found by Smith et al. (2005, see their Fig. 4). This new measurement (the RGB star \#45330) perfectly confirms their finding, adding one new F-rich Na-poor star in this figure. This star could even be the most F-rich RGB ever studied in any GC (in terms of $[\mathrm{F} / \mathrm{Fe}]$ ratios) with $[\mathrm{F} / \mathrm{Fe}]$ almost solar. Moreover, it is interesting to note that this target \#45330 has very close atmospheric parameters and almost the same $\mathrm{F}$ and $\mathrm{Na}$ abundances 
P. de Laverny and A. Recio-Blanco: Fluorine abundances and the puzzle of globular cluster chemical history

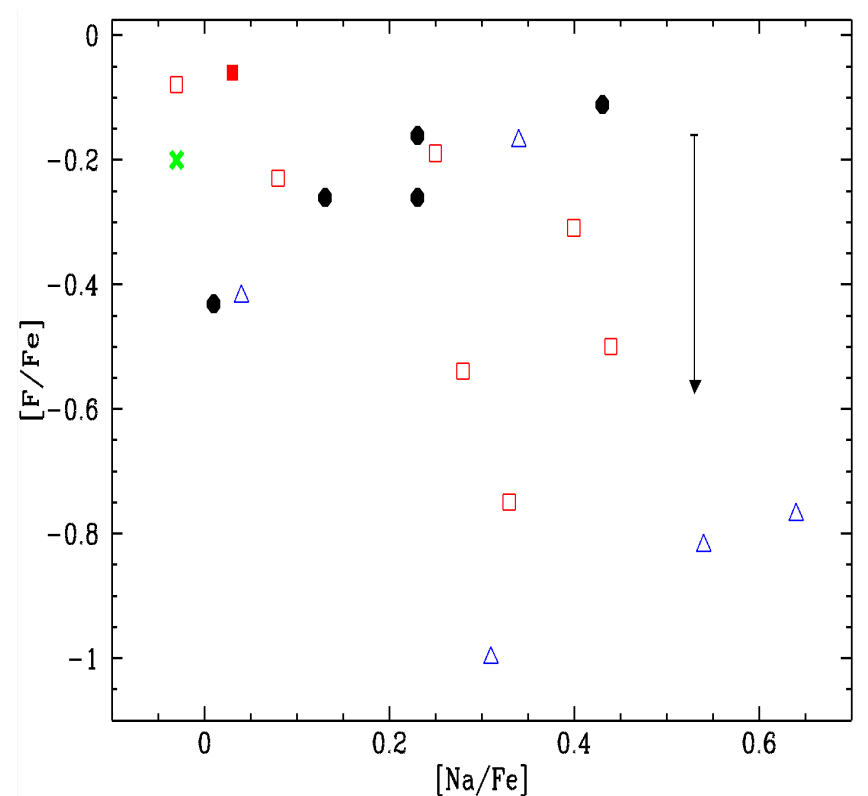

Fig. 3. $[\mathrm{F} / \mathrm{Fe}]$ versus $[\mathrm{Na} / \mathrm{Fe}]$ ratios for RGB stars found in metal-rich or intermediate-metallicity globular clusters: 47 Tuc (this work, filled black circles), M 4 (this work, red filled square and Smith et al. 2005, red empty squares), NGC 6712 (Yong et al. 2008, blue empty triangles) and $\omega$ Cen (Cunha et al. 2003, green cross). The RGB star of 47 Tuc, for which we have derived an upper limit for the fluorine abundance, is indicated with an arrow oriented towards low $[\mathrm{F} / \mathrm{Fe}]$ ratios. The $[\mathrm{F} / \mathrm{Fe}]$ upper limits derived for the stars belonging to the very metal-poor clusters NGC 6397 and M 30 are located well above the upper limit of this plot. Typical errors are around \pm 0.25 dex in both axes.

(within 0.1 dex) as the faintest star analysed by Smith et al. (2005, their star \#L3413).

The two other RGB stars of M 4 analysed in the present study appear to be rather Na-poor, but we have been able to derive only upper limits of their $\mathrm{F}$ content ${ }^{1}$. This is probably caused by the fact that these two stars are not located at the tip of the RGB and are thus rather hot, leading to too weak an HF line to be detected well. Indeed, the only M 4 star for which we have been able to derive a fluorine abundance (\#45330) is about one and two magnitudes brighter in the $K$-band than these two M 4 stars with estimated $\mathrm{F}$ upper limits, respectively. It is also cooler by more than $200 \mathrm{~K}$ than these two fainter targets.

\section{Discussion}

\subsection{Fluorine abundances distribution in metal-rich or intermediate-metallicity globular clusters}

Up to now, fluorine abundances have been safely derived in RGB stars belonging to the metal-rich GC 47 Tuc (this work) and $\omega$ Cen (Cunha et al. 2003) and to the intermediate-metallicity GC M 4 (this work and, mainly, Smith et al. 2005) and NGC 6712 (Yong et al. 2008) ${ }^{2}$. The mean metallicity of these GCs is found to lie between -0.76 and -1.2 dex, and we refer to Sect. 4.2 for the discussion on the fluorine abundance estimates in more metal-poor clusters.

First, we compare in Fig. 3 the $[\mathrm{F} / \mathrm{Fe}]$ and $[\mathrm{Na} / \mathrm{Fe}]$ ratios in these four rather metal-rich GC. In this plot, for the unique

\footnotetext{
1 For clarity reasons, these two stars are not shown in the Fig. 3 described in Sect. 4.1.

2 See the introduction of the present article for a summary of these works.
}

RGB star of $\omega$ Cen with a well defined fluorine abundance i.e. not an upper limit (Cunha et al. 2003), we adopt its sodium abundance derived by Smith et al. (2000). Regarding a possible anticorrelation between fluorine and sodium in GC, it could be seen in this Fig. 3 only for M 4, but the large errors on the abundance ratios could hide different behaviour. Indeed, even in this GC, large F-abundance variations for almost constant Na-abundances are present. For instance, one can find three stars (over a sample of only 8 stars) having almost identical Na-abundances $([\mathrm{Na} / \mathrm{Fe}] \sim 0.25-0.3 \mathrm{dex})$, but their $[\mathrm{F} / \mathrm{Fe}]$ ratios differ by more than 0.6 dex, well beyond the error bars. Such a spread in $\mathrm{F}$ and not in $\mathrm{Na}$ could reveal a rather inhomogeneous chemical history within $\mathrm{M} 4$, particularly regarding the nature of its F-polluters. In any case, such a figure is not consistent with a classical anti-correlation between fluorine and sodium but, owing to the rather large abundance uncertainties, this cannot be concluded definitively.

For the three other clusters, 47 Tuc, NGC 6712 (in which five stars have an estimated F-abundance in each of them), and $\omega$ Cen (only one star), the stars are located into two separated regions of the diagram: F-rich Na-poor and F-poor Na-rich. From their $\mathrm{Na}$ content, it can be claimed that these two groups correspond to the first- and second-generation stars of these GCs, respectively. Moreover, no smooth variation is seen between these two regimes. Indeed, one finds two stars (\#V10 and \#LM10 of Yong et al. 2008) in NGC 6712 with almost identical $[\mathrm{Na} / \mathrm{Fe}]$ but differing by $0.8 \mathrm{dex}$ in $[\mathrm{F} / \mathrm{Fe}]$. It is difficult to claim that any continuous anticorrelation does exist in NGC 6712 when one looks at these two stars. Finally, our RGB targets in 47 Tuc are all located in the F-rich Na-poor part of Fig. 3. It would be nice to see if future studies will report any F-poor Na-rich second-generation stars in this cluster (our target star \#68039 with only an upper limit of its fluorine abundance could be one of these). We recall that three distinct populations have been photometrically found by Milone et al. (2012) in 47 Tuc. Therefore, it is highly probable that $\mathrm{F}$-poor Na-rich ones are present in this cluster.

In summary, with the present situation illustrated in Fig. 3, it can only be said that, except perhaps for M 4, RGB stars in GCs belong to at least two rather well-separated groups in an F-Na diagram: they are either $F$-rich $N a$-poor or $F$-poor $\mathrm{Na}$-rich. Actually, such a dichotomic view cannot be excluded for M 4 if one considers the errors in the $\mathrm{F}$ and $\mathrm{Na}$ abundances. Moreover, in all these GCs, the spread in Na-abundances for the F-rich stars (which all have almost identical $[\mathrm{F} / \mathrm{Fe}]$ around $-0.2 \mathrm{dex}$ ) is rather large (up to $0.5 \mathrm{dex}$ ). It can therefore be concluded that the chemical evolution of fluorine in these clusters is probably more complex than previously claimed and that GCs have probably been formed from very inhomogeneous media since they contain stars with very similar $\mathrm{Na}$ abundances but very different F-abundances and/or rather large F-enrichment with a wide spread in $\mathrm{Na}$.

Finally, it is interesting to point out that the two separated regimes of the F-Na abundances in GC giants could be linked to the separated sequences revealed in colour-magnitude diagrams of most GCs. Such distinct photometric sequences can be opposed to the more continuous ones proposed by most spectroscopic studies. However, such spectroscopic analysis suffer from quite large abundance measurement errors that could hide a multimodal distribution (see for instance, Renzini 2013). Therefore, the two regimes seen in Fig. 3 are indeed consistent with different star formation episodes in globular clusters, thus confirming the photometric description. 


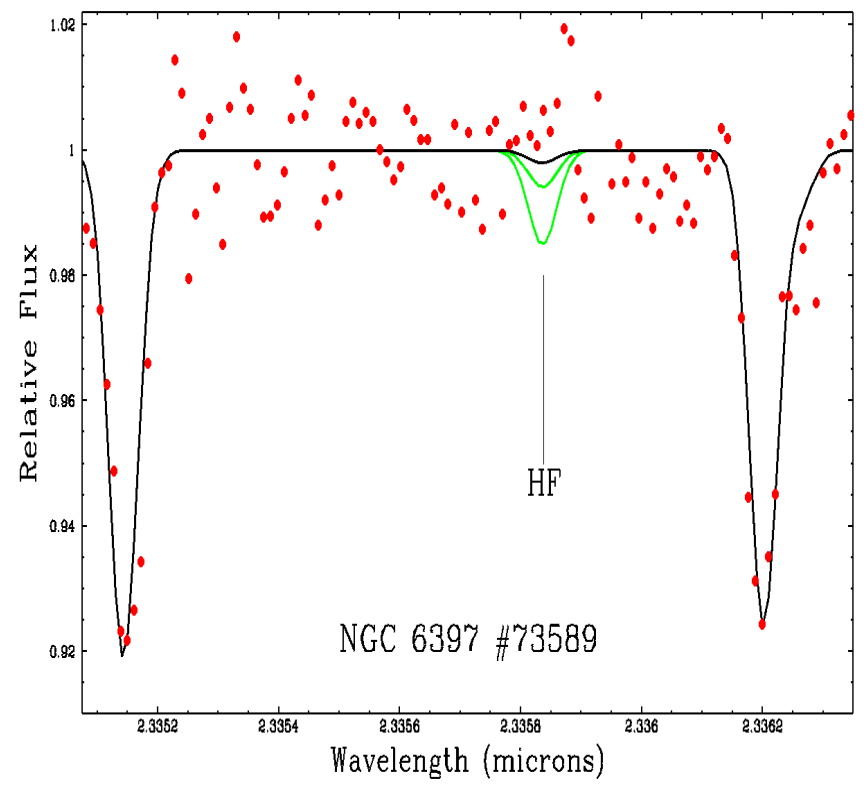

Fig. 4. Observed (filled red circles) and synthetic spectra (continuous lines) around the HF(1-0) R9 line for the star NGC 6397 \#73589. The black line corresponds to a synthetic spectrum computed for the atmospheric parameters of this star reported in Table 1 and adopting the largest fluorine abundance $([\mathrm{F} / \mathrm{Fe}]=-0.1)$ ever measured in any $\mathrm{GC}$. The green lines correspond to synthetic HF lines for a very evolved star at the RGB tip of NGC 6397 with $T_{\text {eff }}=4250 \mathrm{~K}$ and unrealistic F enrichments for a $\mathrm{GC},[\mathrm{F} / \mathrm{Fe}]=-0.1$ and $+0.3 \mathrm{dex}$, respectively.

\subsection{Fluorine in metal-poor globular clusters}

Fluorine abundance studies in metal-poor GC $([\mathrm{Fe} / \mathrm{H}]<$ $\sim-1.5$ dex) have only been reported in NGC 6397 and M 30, which are extremely metal-poor $([\mathrm{Fe} / \mathrm{H}]<-2.0$ dex; present work) and in $\mathrm{M} 22$ with stars having $[\mathrm{Fe} / \mathrm{H}]$ between -1.6 and -1.9 dex (Alves-Brito et al. 2012; D’Orazi et al. 2013).

We have only been able to derive upper limits of the fluorine content in the extreme metal-poor GC NGC 6397 and M 30. These F-abundance upper limits are not shown in Fig. 3 for clarity, since they are much higher than the classical $[\mathrm{F} / \mathrm{Fe}]$ ratios found in more metal-rich clusters. Such a limitation in fluorine determination in metal-poor stars is caused by the possibiliy that, at the CRIRES resolution and even with a rather optimal observing strategy for the telluric standards, the telluric line residuals can have the same amplitude as the HF signature (about $\pm 1 \%$ around the continumm level in the present case). We think that even under optimal and steady weather conditions, it is very difficult to get telluric residuals with an amplitude that is at least twice smaller. Furthermore, metal-poor stars are known to be quite hotter than their metal-rich counterparts. This leads to an even weaker HF line since this molecular transition appears stronger in cooler media, keeping the fluorine abundance constant.

This situation is illustrated in Fig. 4 where it can be seen that the HF line could only be strong enough in a very cool giant star located at the tip of NGC 6397 and having an extreme (and probably not realistic for a GC) F-enrichment. In this figure, we adopt an effective temperature close to the one of the coolest star ever studied in this cluster (see, for instance, Castilho et al. 2000). Of course, the situation is even worse for the even more metalpoor cluster M 30. This figure shows why it has been impossible to report any interesting information on the F-content of these clusters that are too metal-poor.
Futhermore, this problem could be even more complex due to unfortunate radial velocity shifts between the telluric standard and the GC target spectra. Indeed, in some cases, the blue wing of the HF line could be much more contaminated by the telluric residuals than the red one due to the strongest telluric lines found there. This effect leads to an even more difficult Fabundance determination in such metal-poor stars. We think that the above problems can affect the F-abundances in M 22 reported by D'Orazi et al. (2013), even after their revision of the abundance estimates of Alves-Brito et al. (2012). Such an artefact could indeed lead to a wrong identification of the very weak HF line whereas all the other strongest lines $(\mathrm{CO}$ and $\mathrm{Na}$ ) are much less affected by such a wavelength shift (see de Laverny et al., in prep., for a more detailed discussion).

We therefore think that fluorine abundance studies in metalpoor giant stars $([\mathrm{Fe} / \mathrm{H}]<\sim-1.5 \mathrm{dex})$ could face a limitation in performing the correct analysis of the HF line (except if extremely F-enriched stars are encountered as in Schuler et al. 2007). This could only be solved owing to much higher SNR spectra collected under very steady weather conditions and possibly with higher spectral resolution to get a better substraction of the telluric signatures.

\subsection{Comparison of fluorine content in galactic and extragalactic stellar populations}

From Fig. 3 and with the present rather small sample of analysed RGB stars in globular clusters, it can be noted that GC stars are rather weakly enriched in fluorine. Indeed, the derived $[\mathrm{F} / \mathrm{Fe}]$ ratios in these GC stars (with typical values between -0.1 and -1.0 dex) are always much lower than the ones estimated in other samples of galactic ${ }^{3}$ and extragalactic stars.

For instance, bulge stars have $[\mathrm{F} / \mathrm{Fe}]$ ratios in the range $[+0.25,+0.8]$ except one star found with $[\mathrm{F} / \mathrm{Fe}]=-0.27$ (Cunha et al. 2008). The F-abundances have also been estimated in three halo carbon-enriched metal-poor stars that exhibit extremely high $[\mathrm{F} / \mathrm{Fe}]$ ratios (from 0.6 up to 2.9 dex, Schuler et al. 2007; Lucatello et al. 2011). In the galactic disc, members of the Orion Nebula cluster have $[\mathrm{F} / \mathrm{Fe}]$ within -0.05 and +0.1 dex (Cunha $\&$ Smith 2005) and dwarfs of the solar neighbourhood or giants as Arcturus have $[\mathrm{F} / \mathrm{Fe}]$ within +0.0 and +0.55 dex (this work and Recio-Blanco et al. 2012). Moreover, these low [F/Fe] ratios in GC are also much lower than those found in low-mass AGB carbon stars in the solar vicinity in which fluorine is seen to be synthetized and dredged-up to their surface. These lowmass stars are characterized by a $[\mathrm{F} / \mathrm{Fe}]$ ratio between -0.1 and +0.65 dex (Abia et al. 2009, 2010). Only most of the peculiar carbon stars of $\mathbf{J}$ spectral type (which evolutionary origin is still unknown) have slightly negative $[\mathrm{F} / \mathrm{Fe}]$ ratios (between -0.2 and +0.1 dex; Abia et al. 2010), but these ratios are still higher than what is found in most galactic globular cluster giants. Finally, even larger fluorine contents (and probably more efficient insitu production) are found in metal-poor extragalactic carbon stars (Abia et al. 2011). Most of the derived $[\mathrm{F} / \mathrm{Fe}]$ ratios in these low-mass extragalactic AGBs lie between 0.8 and 1.7 dex, whereas their iron content spans the same range of metallicity as the one of the GCs studied in the present work. Such observed large F-enrichments are consistent with theoretical models of low-mass AGB stars that predict higher $[\mathrm{F} / \mathrm{Fe}]$ ratios at lower metallicities.

3 This characteristic has already been partially discussed by Yong et al. (2008) in terms of fluorine abundances in galactic stars but not regarding $[\mathrm{F} / \mathrm{Fe}]$ ratios as in the present work. 
In summary, the observed difference between the $[\mathrm{F} / \mathrm{Fe}]$ ratios estimated in GC stars and in other galactic and extragalactic populations appears to be large (more than a factor 10). This is another confirmation that the chemical evolution history in GCs is quite peculiar with respect to other populations.

\section{Summary}

The IR spectral analysis of 15 stars found in different globular clusters spanning a wide range of metallicity (from metal-rich, $[\mathrm{Fe} / \mathrm{H}]=-0.8$, to extreme metal-poor, $[\mathrm{Fe} / \mathrm{H}]=-2.3$ ) has allowed us to study the fluorine and sodium abundance distributions of these clusters. For the most metal-rich GC of the present study (47 Tuc), we reported the first description of its fluorine content. Fluorine abundances were found to be almost constant (within error bars), whereas sodium strongly varies from starto-star, revealing a rather inhomogeneous cluster. No Na-F anticorrelation was thus seen in 47 Tuc. The only M 4 star for which a fluorine abundance has been derived (forgetting the two other M 4 targets with F upper limits) is consistent with the F-Na distribution already reported by Smith et al. (2005). In the more metal-poor clusters studied (NGC 6397 and M 30), only F-abundance upper limits have been derived with upper values that are too high to provide interesting constraints on the formation history of these clusters. We have indeed shown that fluorine abundances can be safely estimated only in metal-rich or intermediate metallicity clusters. In very metal-poor clusters, exceptional observing conditions and/or very F-enriched stars should be met to derive right estimate of the fluorine abundances. We also pointed out that the chemical history of fluorine in GC seems to strongly differ from other galactic populations found in the disc (dwarfs of the solar neighbourhood, Orion nebula giants, and low-mass AGBs), in the galactic bulge and halo (RGB stars) or in extragalactic dwarf satellites (low-mass metal-poor AGBs).

Finally, in all the clusters studied so far (in the present work or in previous ones), the derived fluorine and sodium abundances showed that RGB stars seem to belong to two well-separated regimes: either F-rich Na-poor or F-poor Na-rich. These two groups are consistent with the first- and second-generation stars found in most GCs. Indeed, no continuous (anti-)correlation between fluorine and sodium abundances are clearly seen in these clusters. It is not seen in 47 Tuc and NGC 6712 and even the anticorrelation reported by Smith et al. (2005) in M 4 could be compatible with these two separate regimes because of the errors in the abundance determinations that could blur such a figure. Such a bimodal distribution is consistent with the separated formation episodes in galactic globular clusters as already revealed by colour-magnitude studies in several GCs. This scenario should be tested by future models of the chemical evolution history of GCs.

\section{References}

Abia, C., Recio-Blanco, A., de Laverny, P., et al. 2009, ApJ, 694, 971

Abia, C., Cunha, K., Cristallo, S., et al. 2010, ApJ, 715, L94

Abia, C., Cunha, K., Cristallo, S., et al. 2011, ApJ, 737, L8

Alonso, A., Arribas, S., \& Martínez-Roger, C. 1999, A\&AS, 140, 261

Alvarez, R., \& Plez, B. 1998, A\&A, 330, 1109

Alves-Brito, A., Yong, D., Meléndez, J., Vásquez, S., \& Karakas, A. I. 2012, A\&A, 540, A3

Cardelli, J. A., Clayton, G. C., \& Mathis, J. S. 1989, ApJ, 345, 245

Carretta, E., Bragaglia, A., Gratton, R., D’Orazi, V., \& Lucatello, S. 2009, A\&A, 508,695

Castilho, B. V., Pasquini, L., Allen, D. M., Barbuy, B., \& Molaro, P. 2000, A\&A, 361,92

Charbonnel, C., Krause, M., Decressin, T., Prantzos, N., \& Meynet, G. 2013, Mem. Soc. Astron. It., 84, 158

Cunha, K., \& Smith, V. V. 2005, ApJ, 626, 425

Cunha, K., Smith, V. V., Lambert, D. L., \& Hinkle, K. H. 2003, AJ, 126, 1305

Cunha, K., Smith, V. V., \& Gibson, B. K. 2008, ApJ, 679, L17

D’Orazi, V., Lucatello, S., Lugaro, M., et al. 2013, ApJ, 763, 22

Gratton, R. G., Carretta, E., \& Bragaglia, A. 2012, A\&ARv, 20, 50

Gustafsson, B., Edvardsson, B., Eriksson, K., et al. 2008, A\&A, 486, 951

Harris, W. E. 1996, AJ, 112, 1487

Hinkle, K., Wallace, L., \& Livingston, W. 1995, PASP, 107, 1042

Jorissen, A., Smith, V. V., \& Lambert, D. L. 1992, A\&A, 261, 164

Lind, K., Charbonnel, C., Decressin, T., et al. 2011, A\&A, 527, A148

Lucatello, S., Masseron, T., Johnson, J. A., Pignatari, M., \& Herwig, F. 2011, ApJ, 729, 40

Milone, A. P., Piotto, G., Bedin, L. R., et al. 2012, ApJ, 744, 58

Momany, Y., Bedin, L. R., Cassisi, S., et al. 2004, A\&A, 420, 605

Pilachowski, C. A., Sneden, C., \& Kraft, R. P. 1996, AJ, 111, 1689

Prantzos, N., Charbonnel, C., \& Iliadis, C. 2007, A\&A, 470, 179

Ramírez, I., \& Meléndez, J. 2005, ApJ, 626, 465

Recio-Blanco, A., de Laverny, P., Worley, C., et al. 2012, A\&A, 538, A117

Renzini, A. 2013, Mem. Soc. Astron. It., 84, 162

Rosenberg, A., Piotto, G., Saviane, I., \& Aparicio, A. 2000, A\&AS, 144, 5

Schuler, S. C., Cunha, K., Smith, V. V., et al. 2007, ApJ, 667, L81

Smith, V. V., Suntzeff, N. B., Cunha, K., et al. 2000, AJ, 119, 1239

Smith, V. V., Cunha, K., Ivans, I. I., et al. 2005, ApJ, 633, 392

Yong, D., Meléndez, J., Cunha, K., et al. 2008, ApJ, 689, 1020 